Int. J. Electrochem. Sci., 13 (2018) 10289 - 10301

\title{
Simple and Sensitive Electrochemical Sensor for Tyramine Determination Based on Overoxidized Poly $(o$-aminophenol) Film Modified Electrode
}

\author{
Xiaojuan Zhao ${ }^{1, *}$, Lijuan $\mathrm{Yi}^{1,2}$, Chunli Wang ${ }^{3}$, Yanping Xian ${ }^{4}$, Xiaofang Zeng ${ }^{1}$, Weidong Bai ${ }^{1}$ \\ ${ }^{1}$ College of Light Industry and Food Science, Zhongkai University of Agriculture and Engineering, \\ Guangzhou 510225, P.R. China \\ ${ }^{2}$ Hotel Management College, Guangzhou Huashang Vocational College, Guangzhou 511300, P.R. \\ China \\ ${ }^{3}$ Jiangmen Entry-Exit Inspection and Quarantine Bureau, Jiangmen 529000, P.R. China \\ ${ }^{4}$ Guangzhou Quality Supervision and Testing Institute, Guangzhou 511447, P.R. China \\ *E-mail: xiao0692@163.com
}

doi: $10.20964 / 2018.11 .43$

Received: 12 June 2018 / Accepted: 14 August 2018 / Published: 1 October 2018

\begin{abstract}
A simple electrochemical sensing method was proposed for fast detection of tyramine in rice vinegar. $o$-Aminophenol (OAP) was electropolymerized on the surface of glassy carbon electrode (GCE) to form the poly $(o$-aminophenol) (POAP) film. Then the overoxidized poly $(o$-aminophenol $)$ film modified GCE (OPOAP/GCE) was obtained by electrochemical treatment of POAP film in the alkaline solution. The modification method of GCE and detection conditions of tyramine were optimized. Electrochemical impedance spectroscopy and scanning electron microscopy were used to characterize the preparation process of OPOAP/GCE and binding ability of tyramine onto the OPOAP film. Furthermore, the electrochemical properties of OPOAP/GCE was investigated by square wave voltammetry. OPOAP/GCE, which was fabricated via cyclic voltammetry in $0.3 \mathrm{M}$ perchloric acid solution with 0.01 M OAP and overoxidized in $0.09 \mathrm{M} \mathrm{NaOH}$ solution, showed good electrochemical response on tyramine. The oxidation current and the concentration of tyramine had good linear relationship in the ranges of $0.1-10 \mu \mathrm{M}$ and $10-200 \mu \mathrm{M}$, respectively. The detection limit was 0.054 $\mu \mathrm{M}$, based on the signal to noise ratio of 3 . The recovery rates of tyramine in rice vinegar sample were from $95.6 \%$ to $117.2 \%$. The sensor was simple, inexpensive, rapid, and suitable to detect tyramine in rice vinegar sample.
\end{abstract}

Keywords: poly( $o$-aminophenol), conducting polymer, electrochemical sensor, tyramine, rice vinegar FULL TEXT 
(C) 2018 The Authors. Published by ESG (www.electrochemsci.org). This article is an open access article distributed under the terms and conditions of the Creative Commons Attribution license (http://creativecommons.org/licenses/by/4.0/). 